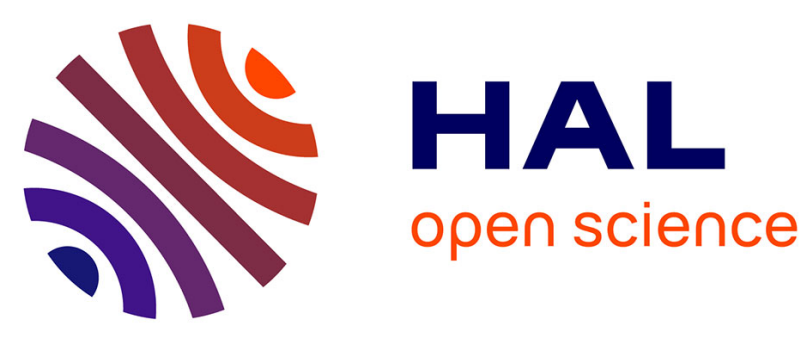

\title{
Sur les phonons d'interface
}

P. Masri

\section{To cite this version:}

P. Masri. Sur les phonons d'interface. Journal de Physique Lettres, 1979, 40 (23), pp.629-632. 10.1051/jphyslet:019790040023062900 . jpa-00231704

\section{HAL Id: jpa-00231704 https://hal.science/jpa-00231704}

Submitted on 1 Jan 1979

HAL is a multi-disciplinary open access archive for the deposit and dissemination of scientific research documents, whether they are published or not. The documents may come from teaching and research institutions in France or abroad, or from public or private research centers.
L'archive ouverte pluridisciplinaire HAL, est destinée au dépôt et à la diffusion de documents scientifiques de niveau recherche, publiés ou non, émanant des établissements d'enseignement et de recherche français ou étrangers, des laboratoires publics ou privés. 


\title{
Sur les phonons d'interface
}

\author{
P. Masri \\ Centre d'Etudes d'Electronique des Solides, \\ Université des Sciences et Techniques du Languedoc, place E.-Bataillon, 34060 Montpellier Cedex, France
}

(Reçu le 15 juillet 1979, révisé le 8 octobre 1979, accepté le 15 octobre 1979)

\begin{abstract}
Résumé. - Nous étudions l'évolution des conditions d'existence et des énergies des phonons optiques d'interface
\end{abstract} solide-solide lorsque l'épaisseur de l'un des deux cristaux varie.

Abstract. - We study the evolution of the existence conditions and energies of optical phonons of a solid-solid interface when the thickness of one of the two crystals changes.

1. Introduction. - L'interface entre deux cristaux différents présente un intérêt technologique bien connu : c'est le cas des jonctions métal-semiconducteur (diodes) et des hétérojonctions (jonctions entre deux semiconducteurs différents). Les études s'y rapportant sont stimulées par les progrès techniques dans ce domaine : on peut citer la technique d'épitaxie par jets moléculaires (MBE) [1] qui permet d'obtenir une croissance contrôlée couche par couche. Les structures vibrationnelles spécifiques d'une interface peuvent jouer un rôle important dans plusieurs propriétés physiques. Ainsi les mesures de mobilité électronique dans le cas de surfaces de silicium couvertes de couches d'oxyde $\left(\mathrm{SiO}_{2}\right)$ [2] font ressortir le rôle important des phonons optiques dans le mécanisme de diffusion des électrons à des températures supérieures à $150 \mathrm{~K}$. L'on sait que le couplage phononélectron intervient dans les propriétés électriques de l'hétérojonction : d'où l'intérêt de connaître les caractéristiques vibrationnelles spécifiques du dispositif. L'adsorption de gaz rares (Ar, $\mathrm{Ne}$ ) sur des couches minces $(\mathrm{Al}, \mathrm{Sn} . .$.$) produit une diminution$ de la température de transition vers l'état supraconducteur [3]. Les résultats sont interprétés comme dus à la modification du spectre des phonons de la couche mince par la présence des atomes de gaz rares. L'existence de modes localisés a aussi une influence sur la pression de vapeur dans des expériences de condensation sur substrat solide : les atomes adsorbés restent dans un état correspondant à une énergie d'activation de sublimation influencée par la présence de phonons localisés à l'interface [4].

(*) Associé au CNRS (LA 21).
Dans les expériences de collision atome-solide, la présence de modes localisés à l'interface a une influence sur les caractéristiques de la collision.

Du point de vue expérimental, différentes techniques sont utilisées pour étudier les caractéristiques vibrationnelles des défauts (surfaces, adsorption...) : spectroscopie électronique par pertes d'énergie $[5,6]$, diffusion inélastique des neutrons [6], diffusion Raman [6], spectroscopie infrarouge [6], diffusion Brillouin [7].

$\mathrm{Si}$ les propriétés vibrationnelles caractéristiques d'une monocouche adsorbée [8] et d'une interface entre deux solides semi-infinis différents $\mathbf{A}$ et $\mathbf{B}$ [9] ont déjà été abordées, il n'existe pas, par contre, d'études comparatives montrant l'évolution de ces propriétés au cours de la croissance du cristal B sur le cristal $\mathrm{A}$. Ce travail en constitue une première approche et concerne seulement le cas des phonons du type optique. Nous le présentons dans le cadre du modèle de dynamique de réseau le plus simple possible, le modèle unidimensionnel, dans lequel on assimile le cristal à un ensemble de chaînes linéaires. Comme nous nous intéressons aux phonons optiques, la faiblesse de ce modèle due au fait que l'énergie potentielle élastique qui lui est associée n'est pas invariante par rotation infinitésimale de l'ensemble du cristal n'est pas catastrophique tant que l'on n'aborde pas le cas des phonons acoustiques. D'autre part, il a été prouvé [10] que ce modèle donnait des résultats comparables à ceux d'un modèle tridimensionnel dans le cas de l'adsorption [11]. Enfin, il donne des modes d'interface, entre deux cristaux différents, du type optique, comme dans le cas d'un modèle tridimensionnel. Par contre il permet une étude 
analytique dont le seul objet est de mettre en évidence les effets physiques auxquels l'on doit s'attendre dans le cadre d'une étude utilisant des modèles plus réalistes.

Dans ce qui suit, nous présentons les résultats concernant l'évolution des conditions d'existence des modes d'interface dans les cas de l'interface entre un cristal $A$ et : $1 \mathrm{M}$,

- une monocouche d'atomes B adsorbés : modèle - deux monocouches d'atomes B adsorbés : modèle $2 \mathrm{M}$,

- un cristal B semi-infini.

Nous montrons aussi comment varient les énergies des phonons caractéristiques de l'interface au cours de la croissance de $B$.

2. Evolution des conditions d'existence des modes d'interface en fonction de l'épaisseur de la couche B. Nous désignerons par $K_{\mathrm{i}}$ la constante de force à l'interface, $\left(K_{\mathrm{A}}, M_{\mathrm{A}}\right)$ et $\left(K_{\mathrm{B}}, M_{\mathrm{B}}\right)$ les constantes des forces centrales entre deux atomes premiers voisins et les masses atomiques respectivement dans les deux cristaux A et B. Nous supposerons que le cristal A a la plus faible pulsation maximale en volume : $\omega_{\mathrm{mA}}<\omega_{\mathrm{mB}}$. Nous ne tenons pas compte des variations éventuelles des constantes de force au voisinage de l'interface.

2.1 CAS D'Une MONOCOUChe ADSORBÉE. MODÈLE 1 M. - Dans ce cas, l'on sait [12] qu'il existe un mode localisé à l'interface du type optique caractéristique de la monocouche adsorbée dans des conditions telles que :

$$
\beta>\frac{4 \mu}{2 \mu+1}
$$

avec

$$
\beta=\frac{K_{\mathrm{i}}}{K_{\mathrm{A}}} \quad \text { et } \quad \mu=\frac{M_{\mathrm{B}}}{M_{\mathrm{A}}} \text {. }
$$

Au-dessus de la courbe $\beta_{\mathrm{L}}=\frac{4 \mu}{2 \mu+1}$ (fig. 1) on a un mode localisé du type optique. En dessous, ce mode n'existe plus. Ce résultat signifie que le mode localisé apparaît lorsque l'interaction à l'interface $\left(K_{\mathrm{i}}\right)$ est suffisamment forte pour qu'il sorte par le haut de la bande de volume du cristal A, l'effet d'un accroissement de $K_{\mathrm{i}}$ se traduisant par une augmentation des pulsations.

2.2 CAS DE « DEUX MONOCOUCHES ADSORBÉES 》. Modèle $2 \mathrm{M}$. - Nous nous limiterons au cas où $K_{\mathrm{B}}=K_{\mathrm{A}}$ pour lequel le plan $(\beta, \mu)$ se divise en trois régions [10] délimitées par les courbes (1) et (2) (voir fig. 1) données par les équations suivantes :

$$
\frac{8 \mu(2 \mu-1)}{8 \mu^{2}-1}=\left\{\begin{array}{l}
\beta_{1}(\text { courbe } 1) \text { pour } 0 \leqslant \mu<8^{-1 / 2} \\
\beta_{2}(\text { courbe } 2) \text { pour } 8^{-1 / 2}<\mu \leqslant 1 .
\end{array}\right.
$$

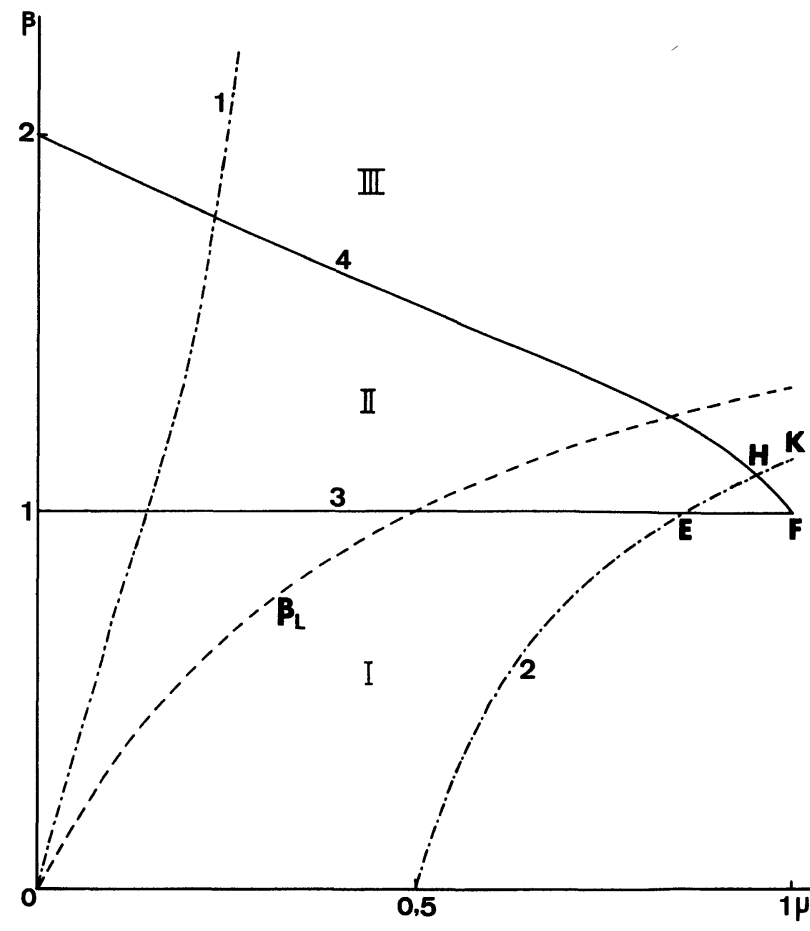

Fig. 1. - Evolution des conditions d'existence des phonons d'interface avec l'épaisseur du cristal $B$ en fonction de $\mu$ dans le cas où $K_{\mathrm{A}}=K_{\mathrm{B}}$.

[Evolution of existence conditions of interface phonons with the thickness of B crystal in function of $\mu$ for $K_{\mathrm{A}}=K_{\mathrm{B}}$.]

En dessous de $\beta_{2}$ nous n'avons pas de modes localisés d'interface. Lorsque $K_{\mathrm{i}}$ augmente, un mode localisé $\left(\beta_{2}<\beta<\beta_{1}\right)$ ou deux $\left(\beta>\beta_{1}\right)$ peuvent apparaître.

Lorsque nous passons d'une monocouche à deux, il apparaît donc un nouveau domaine délimité par l'axe $\mu, \beta_{2}$ et $\beta_{\mathrm{L}}$ pour lequel on a un mode localisé. Un premier effet est donc mis en évidence : à $\eta=K_{\mathrm{B}} / K_{\mathrm{A}}$ et $\mu$ donnés, l'adsorption d'une deuxième monocouche favorise l'apparition d'un mode localisé pour des interactions à l'interface $\left(K_{\mathbf{i}}\right)$ moins fortes que celles requises pour obtenir un tel mode avec une monocouche. Un deuxième effet est l'apparition d'un second mode d'interface pour des valeurs suffisamment élevées de $K_{\mathrm{i}}$.

2.3 CAS LIMITE DE DEUX CRISTAUX SEMI-INFINIS. Dans ce cas l'étude des conditions d'existence des modes d'interface ainsi que le calcul de leurs énergies peuvent se faire à l'aide de la méthode des fonctions de Green [9].

$\mathrm{Si} \mathbf{G}_{\mathbf{S}}^{\mathrm{A}}$ et $\mathbf{G}_{\mathbf{s}}^{\mathrm{B}}$ désignent les fonctions de Green associées respectivement aux deux cristaux semiinfinis $A$ et $B$, et si $\mathbf{V}_{\mathbf{i}}$ désigne la matrice de perturbation due à la création de l'interface en collant les deux cristaux par une interaction $K_{\mathbf{i}}$, toutes les informations dont on a besoin sont obtenues à partir du déterminant : $D_{\mathbf{i}}=$ dét $\left(\mathbf{I}-\mathbf{V}_{\mathbf{i}} \mathbf{G}_{\mathrm{s}}\right)$ où $\mathbf{I}$ est la matrice unité. Dans le cadre du modèle d'interaction à forces 
centrales entre atomes premiers voisins utilisé ici, $V_{i}$ s'écrit :

où

$$
\mathbf{V}_{\mathrm{i}}=\frac{K_{\mathrm{i}}}{M_{\mathrm{A}}}\left[\begin{array}{l}
1-\sqrt{\theta+1} \\
-\sqrt{\theta+1} \quad \theta+1
\end{array}\right]
$$

On exprime les éléments de la matrice $\mathbf{G}_{\mathrm{s}}$ en fonction de ceux de la matrice $\mathbf{G}_{0}$ associée au cristal parfait au moyen de la relation de Dyson :

$$
\mathbf{G}_{\mathrm{s}}=\mathbf{G}_{0}+\mathbf{G}_{0} \mathbf{V}_{\mathrm{s}} \mathbf{G}_{\mathrm{s}}
$$

où $\mathbf{V}_{\mathrm{s}}$ est la matrice de perturbation due à la création d'une surface libre

$$
\mathbf{V}_{\mathrm{s}}=\frac{K}{M}\left[\begin{array}{rr}
-1 & 1 \\
1 & -1
\end{array}\right]
$$

On obtient :

$$
\begin{aligned}
G_{\mathrm{s}}\left(l, l^{\prime}\right)=G_{0}\left(l-l^{\prime}\right)- & \frac{K}{M}\left[G_{0}(l)-G_{0}(l-1)\right] \times \\
& \times\left[G_{\mathrm{s}}\left(0, l^{\prime}\right)-G_{\mathrm{s}}\left(1, l^{\prime}\right)\right]
\end{aligned}
$$

où l'on ne s'intéresse qu'à $l$ et $l^{\prime} \geqslant 1$.

$G_{0}(l)$ est donnée par l'expression suivante [13] :

$$
G_{0}(l)=\frac{M}{K} \frac{t^{1}+|l|}{t^{2}-1} .
$$

Dans le cristal coupé en deux pour créer une surface libre, on n'a plus de propagation entre les deux demi-cristaux ainsi obtenus, d'où :

$$
G_{\mathrm{s}}\left(0, l^{\prime}\right)=0 \text { pour } l^{\prime} \geqslant 1 .
$$

On obtient alors l'expression suivante du déterminant $D_{\mathrm{i}}$ :

$$
D_{\mathrm{i}}\left(\omega^{2}+\mathrm{i} \varepsilon\right)=1-\frac{K_{\mathrm{i}}}{K_{\mathrm{A}}} \frac{t_{\mathrm{A}}}{t_{\mathrm{A}}-1}-\frac{K_{\mathrm{i}}}{K_{\mathrm{B}}} \frac{t_{\mathrm{B}}}{t_{\mathrm{B}}-1} .
$$

Les valeurs des paramètres pour lesquelles l'équation Réel $D_{\mathrm{i}}=0$ est vérifiée pour $\omega_{\mathrm{mA}}<\omega<\omega_{\mathrm{mB}}$ ou $\omega>\omega_{\mathrm{mB}}$ définissent respectivement les domaines d'existence des modes semi-localisés et localisés. Les courbes limites correspondantes ont pour équation :

$$
\begin{aligned}
& \beta_{3}=\frac{2}{1+1 / \eta} \\
& \beta_{4}=\frac{2(1+1 / \eta)+2\left[(1+1 / \eta)^{2}-1 / \eta(\mu+2+1 / \eta)\right]^{1 / 2}}{1 / \eta(\mu+2+1 / \eta)} .
\end{aligned}
$$

Nous avons représenté sur la figure 1 les trois domaines ainsi obtenus pour $\eta=1$ ( $\beta_{3}$ courbe $3, \beta_{4}$ courbe 4$)$ pour $\mu$ variant de 0 à $1\left(\omega_{\mathrm{mA}} \leqslant \omega_{\mathrm{mB}}\right)$. Dans le domaine I (en dessous de la courbe 3 ) il n'existe pas de mode d'interface. Le long de la courbe 3 on a un mode résonnant dans la partie commune aux deux bandes (résultat déjà obtenu à l'aide d'un modèle plus réaliste [9]). Entre les courbes 3 et 4 (domaine II) il existe un mode d'interface semi-localisé. Au-dessus de la courbe 4 (domaine III) on a un mode localisé à l'interface.

L'évolution de ces domaines des modèles $1 \mathrm{M}$, $2 \mathrm{M}$ à celui pour lequel l'épaisseur de la couche $\mathrm{B}$ devient très grande montre l'effet suivant : les modes localisés éventuels peuvent persister, donner des modes semi-localisés ou bien disparaître. Lorsque les caractéristiques des deux milieux sont voisines ( $\mu$ voisin de $1, \omega_{\mathrm{mA}}$ voisin de $\omega_{\mathrm{mB}}$ ) et dans des conditions telles qu'il n'existait pas de modes d'interface dont le modèle $2 \mathrm{M}$ (fig. 1, domaine EFK), il peut apparaître soit un mode semi-localisé (domaine EFH) soit un mode localisé (domaine FHK) à l'interface.

Avec les valeurs suivantes des paramètres : $\eta=\frac{1}{1,9} ; \mu=\frac{1}{2,4} ; \beta=0,947$ nous obtenons avec le modèle unidimensionnel un mode localisé à l'interface de pulsation $\omega_{\mathrm{L}} / \omega_{\mathrm{mB}} \simeq 1,01$ en accord avec les résultats de la référence [9].

3. Evolution de l'énergie des modes d'interface en fonction de l'épaisseur de la couche $B$. - Au cours de la croissance, le diagramme d'énergie des phonons dans le cristal B se construit progressivement. Ces phonons vont alors interagir avec ceux localisés à l'interface, s'ils existent, ainsi qu'avec ceux du cristal A, pour en modifier respectivement l'énergie et la densité. Ces modes localisés peuvent alors disparaître si leur énergie se mélange avec celles des phonons de volume des deux cristaux, se transformer en modes semilocalisés ou localisés à l'interface.

Pour $\eta=1, \mu=0,5$ on a un mode localisé avec le modèle $1 \mathrm{M}$ (fig. $2 a$ courbe 1 ) pour $\beta>1$. L'adsorption d'une deuxième monocouche va étendre vers les faibles valeurs de $\beta$ le domaine d'existence du mode localisé (courbe 2 ) qui existe maintenant dès que $\beta$ est non nul et dont la pulsation est plus élevée que dans le cas $1 \mathrm{M}$. Lorsque le cristal $\mathrm{B}$ devient très épais ce dernier mode peut :

- disparaître pour $0<\beta \leqslant 1$

- se transformer en un mode semi-localisé à l'interface (courbe 3 ) de pulsation plus basse pour $1<\beta<1,475$ ou plus élevée pour $1,475<\beta<1,545$,

- se transformer en un mode localisé à l'interface de pulsation supérieure pour $\beta>1,545$ (courbe 4).

Les résultats obtenus pour des épaisseurs allant de trois (3 M) à vingt et une monocouches (21 M) montrent que l'énergie maximale $\left(\hbar \omega_{\mathrm{mB}}\right)$ des phonons caractéristiques du cristal massif $B$ n'est atteinte que pour des épaisseurs supérieures à vingt et une monocouches (voir fig. $2 a$ ) et que l'établissement de cette structure vibrationnelle de volume $\left(\omega_{\mathrm{mB}}\right)$ est une condition nécessaire pour l'établissement du mode semi-localisé (courbe 3). Par contre l'énergie 

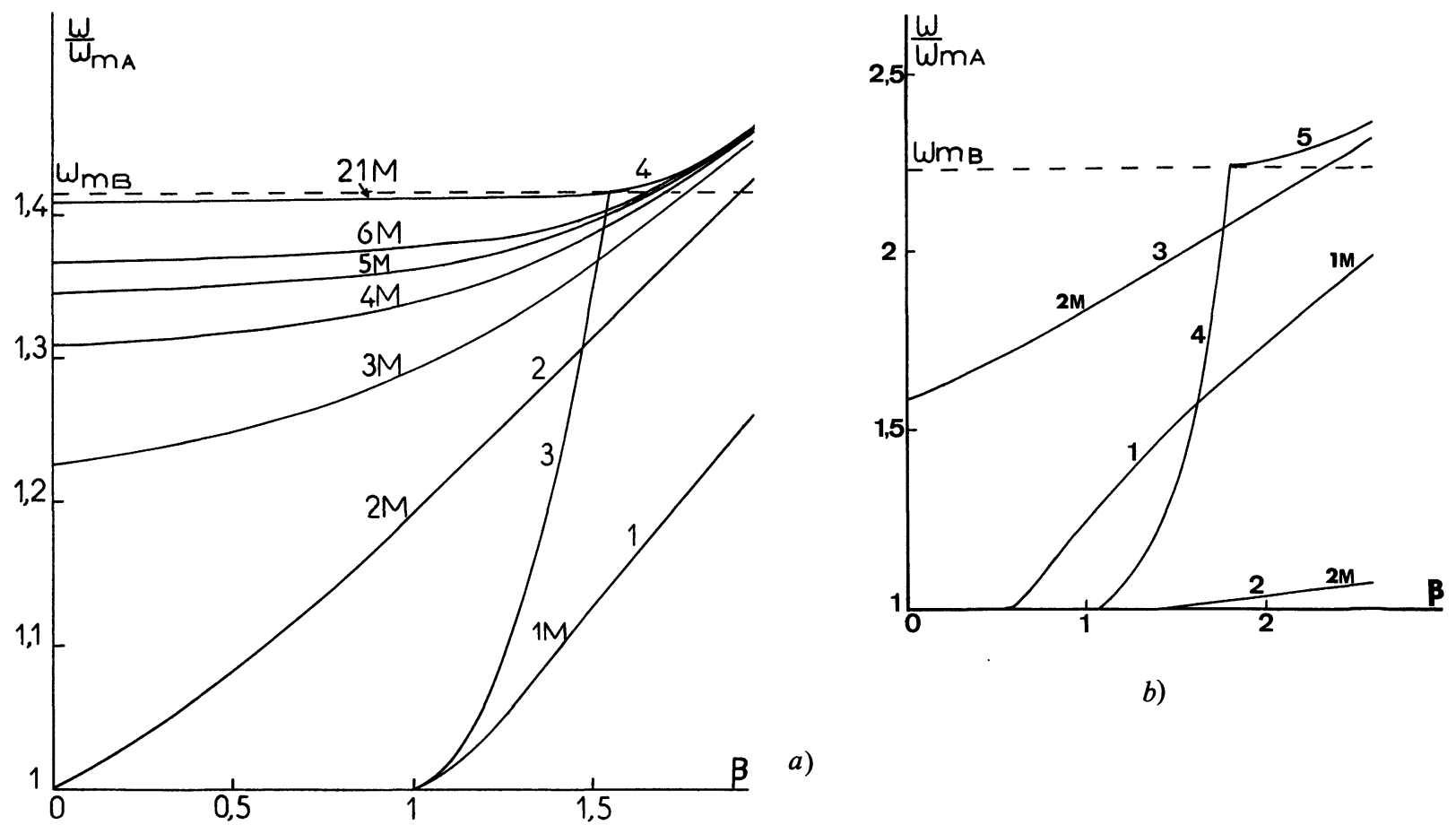

b)

Fig. 2. - Evolution des énergies des phonons d'interface avec l'épaisseur du cristal B pour différentes valeurs de $\beta: a) K_{\mathrm{A}}=K_{\mathrm{B}}, M_{\mathrm{A}}=2 M_{\mathrm{B}}$; b) $K_{\mathrm{A}}=K_{\mathrm{B}}, M_{\mathrm{A}}=5 M_{\mathrm{B}}$.

[Evolution of interface phonons energies with the thickness of B crystal for several values of $\left.\beta: a) K_{\mathrm{A}}=K_{\mathrm{B}}, M_{\mathrm{A}}=2 M_{\mathrm{B}} ; b\right) K_{\mathrm{A}}=K_{\mathrm{B}}$, $M_{\mathrm{A}}=5 M_{\mathrm{B}}$.]

du phonon d'interface (courbe 4) est pratiquement atteinte avec le modèle à $21 \mathrm{M}$.

Il faut noter l'effet suivant : la pulsation du mode localisé à l'interface dans le cas $2 \mathrm{M}$ (courbe 2) est d'autant plus voisine de celle correspondante au cristal B épais (courbe 4) que l'interaction $K_{\mathrm{i}}$ à l'interface est forte; les caractéristiques de l'interface relatives aux phonons optiques sont alors atteintes dès les premières couches de croissance.

Avec $\eta=1, \mu=0,2$ nous obtenons un mode localisé avec le modèle $1 \mathrm{M}$ (fig. $2 b$ courbe 1 ) existant pour $\beta>0,58$. L'adsorption d'une deuxième monocouche le transforme en un mode localisé de pulsation plus élevée (courbe 3 ) qui existe dès que $\beta>0$. Il apparaît aussi, à des pulsations plus basses, un deuxième mode localisé (courbe 2) qui n'existe que pour $\beta>1,41$. Le mode de plus haute énergie (courbe 3 ) est plus sensible à la constante de force à l'interface $K_{\mathrm{i}}$. Lorsque $\mathrm{B}$ devient très épais, ce mode se transforme en un mode d'interface (courbe 5) pour $\beta>1,81$; il peut aussi exister dans ce cas un mode semi-localisé pour $1<\beta<1,81$.

4. Conclusion. - Nous avons présenté pour la première fois une étude de l'évolution des conditions d'existence et des énergies des phonons optiques caractéristiques d'une interface entre un cristal A et une couche B lorsque l'épaisseur de B varie. Plusieurs effets ont été ainsi mis en évidence. Bien que le modèle utilisé ici soit simple, nous pensons qu'ils sont qualitativement représentatifs des tendances qui peuvent apparaître avec des modèles plus réalistes.

Remerciement. - Je remercie vivement le Professeur J. Friedel qui, par son intérêt pour ce travail, m'a encouragé à le publier.

\section{Bibliographie}

[1] Foxon, C. T., Acta Electron. 21 (1978) 139

[2] SAH, C. T., Ning, T. H. et TsChOPP, L. L., Surf. Sci. 32 (1972) 561.

[3] Naugle, D. G., Baker, J. W. et Allen, R. E., Phys. Rev. B 7 (1973) 3028

[4] Levenson, L. L., Thèse Université Paris-Sud Orsay, $n^{\circ}$ d'ordre 387, juin 1968.

[5] IвасH, H., Phys. Rev. Lett. 27 (1971) 253 et 41 (1978) 958.

[6] Voir dans Proc. Int. Conf. Vibrations in Adsorbed Layers (Julich, juin 1978) : IBACH, H., Nielsen, M., HoRN, H.

[7] Bortolani, V., Nizzoli, F., Santoro, G., Marvin, A. et SANDercock, J. R., Phys. Rev. Lett. 43 (1979) 224.
[8] Voir par exemple MasRi, P. et Dobrzynski, L., J. Phys. Chem. Solids 34 (1973) 847.

[9] MasRi, P. et Dobrzynski, L., Surf. Sci. 34 (1973) 119. Duafari-Rouhani, B., Masri, P., Dobrzynski, L., Phys. Rev. B 15 (1977) 5690.

[10] Masri, P. et Armand, G., Surf. Sci. 19 (1970) 53.

[11] Grimley, T. B., Proc. Phys. Soc. (London) 79 (1962) 1203.

[12] Wallis, R. F., Surf. Sci. 2 (1964) 146.

[13] Dobrzynski, L. et Milis, D. L., J. Phys. Chem. Solids 30 (1969) 1043. 